\title{
Discriminazioni di ieri e di oggi: il retaggio del colonialismo
}

\author{
di Chiara Volpato
}

Feb 19, 2021 | Emergenza e (nuova) normalità, In evidenza, Uguaglianza/Differenza | $\underline{0}$ |

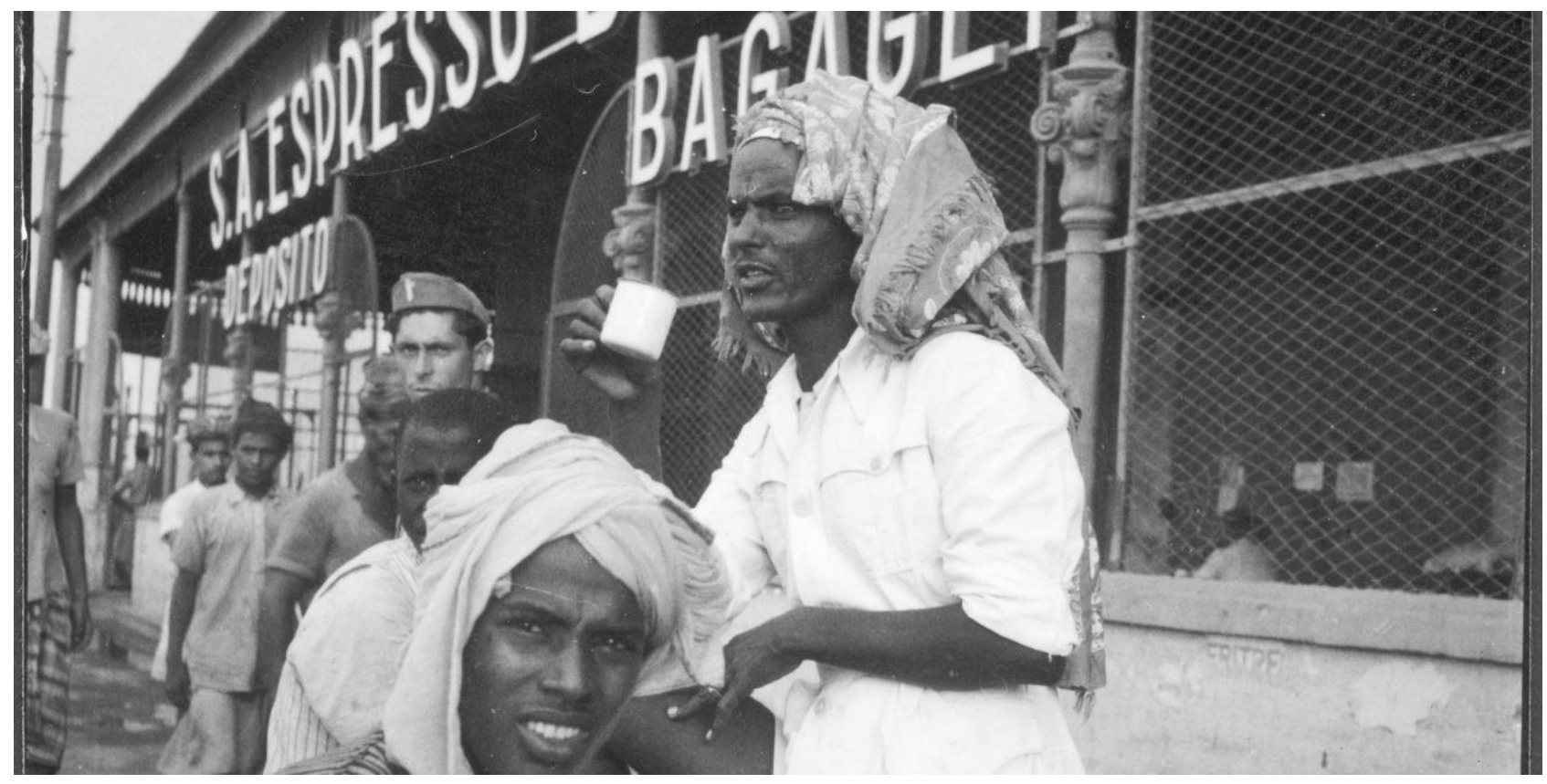

Porto di Massaua (Eritrea), 1939.

Foto di Annemarie Schwarzenbach - https://www.helveticarchives.ch/detail.aspx?ID=550265, Pubblico dominio, Collegamento

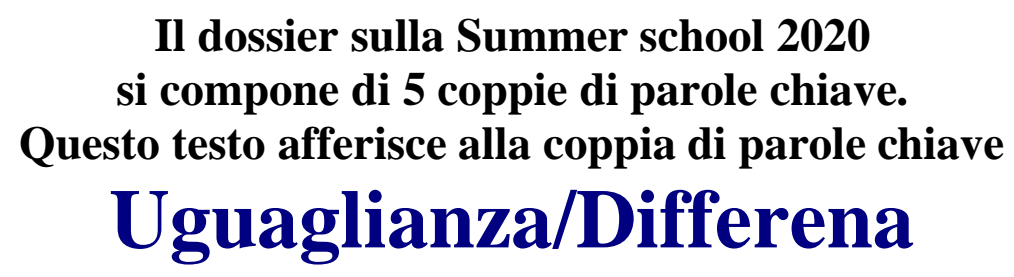

Abstract

Nella seconda giornata della Summer School 2020 abbiamo chiesto a Enrico Manera e Chiara Volpato di riflettere con noi sulle parole chiave Uguaglianza e Differenza. Chiara Volpato ci aiuta con questo suo intervento a individuare quanto ci sia di ricollegabile al passato coloniale negli attuali episodi di discriminazione sociale, anche aggressiva, che si verificano nel nostro Paese. Un passato peraltro oggetto di una tenace rimozione collettiva, che non ha certo impedito il riaffiorare, in contesti di emergenza, di antichi stereotipi culturali razzisti e fascisti, a loro volta alimentati dalle nuove pulsioni nazionaliste e identitarie.

\section{Pandemia, biologizzazione e deumanizzazione}

La pandemia e le misure prese per contrastarla hanno provocato in molte persone sentimenti di solitudine, esclusione, percezione di allentamento o vera e propria perdita dei legami sociali; 
sentimenti destinati a durare dato che alcuni vincoli alla socialità consueta sembrano diventare permanenti e ancora oggi facciamo fatica a intravedere una fine delle restrizioni. Tutto questo ha causato il dilagare di stati d'animo di ansia, angoscia, incertezza, paura di perdere il controllo sulla propria esistenza, stati negativi che - la storia ci insegna ${ }^{[1]}$ - possono facilmente dare adito ad atteggiamenti e comportamenti di rabbia, disperazione, tendenza a cercare facili capri espiatori.

Nei giorni iniziali della pandemia sono emersi alcuni segnali in questo senso; si pensi agli episodi di pregiudizio e discriminazione contro persone cinesi, verificatisi in Italia e in altri paesi; i cinesi sono stati, in casi per fortuna isolati, trattati come "untori" e sono divenuti oggetto di processi di biologizzazione, una particolare forma di de-umanizzazione, che fa percepire l'altro come un contagio, un virus, una peste.

Le metafore biologizzanti, che affondano le radici nel culto della limpieza de sangre della Spagna cinquecentesca, hanno conosciuto un ampio sviluppo soprattutto nel corso dell'Ottocento, in parallelo con l'affermazione del razzismo scientifico e del darwinismo sociale; sono collegate ai concetti di malattia, protezione dell'igiene, purezza e trasformano l'altro, il diverso, in microbo, virus, pestilenza, cancro, sporcizia, inquinamento. Dall'Illuminismo in poi, la teoria dei germi ha sostituito il demonio come metafora di un pericolo oscuro, da affrontare con misure drastiche, come quelle poste in atto di fronte a emergenze ed epidemie, quindi attraverso pratiche di pulizia, eliminazione, disinfezione, purificazione, alle quali si sono ispirati gli autori di tutti i genocidi del Novecento. Hitler, ad esempio, nel Mein Kampf de-umanizzava ossessivamente i gruppi nemici: per descrivere ebrei e marxisti, accanto al registro animale (iene, serpenti, vipere), impiegava il registro biologico (pestilenza, malattia, virus, intossicazione, contagio, infezione). Gli ebrei in particolare venivano raffigurati come: parassiti, bacilli, microbi dannosi, cancro che infetta il corpo dell'umanità ${ }^{[2]}$. Anche le pagine della Difesa della Razza, rivista di propaganda fascista pubblicata dal 1938 al 1943, testimoniano il continuo paragone tra ebrei e animali (avvoltoi, topi, serpi), demoni (diavoli, uccisori di Dio), elementi patologici (bacilli, piaga, morbo, veleno). ${ }_{[}^{[3]}$

Nel corso della pandemia, comunque, la discriminazione verso i cinesi ha perso di intensità perché siamo divenuti tutti, metaforicamente, virus e contagi, come è risultato da un'indagine effettuata con alcuni colleghi delle università di Milano-Bicocca e di Genova, che ha documentato, per la prima volta nella letteratura psicosociale, un processo di biologizzazione relativo al gruppo di appartenenza. ${ }^{[4]}$ Ha prevalso nella popolazione il senso di combattere una battaglia comune (per usare una metafora abusata da politici e media) e, come succede in tutti i conflitti, la condivisione del pericolo ha favorito il senso di coesione e unità.

Durante l'ondata autunnale, però, atteggiamenti e comportamenti aggressivi sono riemersi con forza, a causa probabilmente del sentimento di frustrazione dovuto al ritorno del contagio, delle sofferenze ad esso connesse e delle preoccupazioni legate alla situazione economica. Si è così innescato un fenomeno ben conosciuto dagli scienziati sociali: la ricerca di capri espiatori, individuati in questa occasione nel personale sanitario. Secondo quanto segnalato dai mass media ${ }^{[5]}$, in Lombardia un quarto dei medici si è sentito vittima di discriminazione sociale a causa del proprio impegno contro il Covid 19. In molti casi medici e infermieri sono stati insultati e minacciati per il semplice fatto di denunciare i rischi del contagio e quanto accade all'interno degli ospedali.

Gli atteggiamenti e i comportamenti aggressivi emersi verso tali improbabili capri espiatori costituiscono, a mio parere, un indizio da non sottovalutare, che ci dice che tali episodi potrebbero ripetersi in modo più grave se le angosce derivanti dalla situazione economica dovessero aggravarsi. 


\section{Colonialismo italiano e violenza di genere}

Tra i fenomeni di discriminazione, anche violenta, cui troppo spesso assistiamo oggi - e del resto non certo generati dalla pandemia - c'è quello che riguarda le donne immigrate. Esso, a mio parere, va collegato, per essere pienamente compreso, alle violenze cui sono state sottoposte le donne africane durante la colonizzazione. Tale collegamento è, a mio parere, acuito dalla mancata rielaborazione del passato coloniale italiano e di ciò che tale passato ha significato per la coscienza civile del nostro paese.

L'avventura coloniale italiana è stata fondamentalmente maschile. Nelle colonie si sono recati in grandissima maggioranza uomini, con ruoli diversi e complementari: esploratori, missionari, soldati, dirigenti, operai, contadini. Le donne italiane in Africa sono state rare apparizioni; la loro presenza si è intensificata solo negli anni successivi alla conquista dell'Etiopia quando il regime incoraggiò il trasferimento di famiglie di militari e coloni; tale operazione fu però di breve durata, dato che nell' aprile del 1941 gli inglesi entrarono ad Addis Abeba, rimettendo sul trono il négus Hailé-Selassié I. [6]

Le colonie furono quindi luoghi essenzialmente maschili, in cui il sessismo italiano poté dispiegare i suoi toni più espliciti e volgari, senza le poche remore presenti in madrepatria. Per la sua stessa essenza, la colonia moltiplicava il "dividendo patriarcale", vale a dire il vantaggio che gli uomini ottengono dalla subordinazione delle donne, vantaggio che si esprime attraverso divari di reddito, ma anche di onore, prestigio, autorità. Il Duce stesso, in un discorso, parlò della guerra per la conquista dell'Etiopia come di «una prova che collauda la virilità del popolo Italiano». ${ }^{[7]}$ Del resto, $\mathrm{i}$ primi e i più accesi sostenitori delle imprese coloniali erano stati i gruppi nazionalisti, che si distinguevano anche per le posizioni antifemministe (mentre i primi gruppi femministi di indirizzo radicale ed emancipazionista si erano con decisione opposti all'avventura coloniale, l'Associazione per la donna fu addirittura sciolta nel 1898 per essersi schierata in questo senso). Con il tempo però le cose cambiarono. Nel 1911, in un clima culturale in cui il nazionalismo era ormai egemone, alcune femministe sostennero l'invasione della Libia, cosa che costituì la prima frattura del movimento delle donne.

Lo scenario coloniale divenne così il luogo ideale in cui era lecito scaricare le pulsioni aggressive camuffate in patria; la lontananza dall'Europa permetteva di dare libero sfogo a comportamenti di estrema violenza nei confronti dei resistenti all'occupazione e di estremo machismo verso le native, che, in quanto suddite, erano tenute a farsi carico dei "bisogni" degli occupanti italiani. Gli uomini africani furono raffigurati quali oziosi nullafacenti, da sfruttare come manodopera di bassa lega, o come sciftà, briganti, da eliminare. Le donne furono invece dipinte come prede facili e dissolute per gli appetiti sessuali dei conquistatori.

La propaganda che accompagnò la colonizzazione italiana si servì di una retorica erotizzante, in linea con la cosiddetta porno-tropics tradition, imperniata sulla metafora della Venere nera, che sottolineava i tratti esotici ed erotici delle donne africane, riconoscendo loro la sola identità sessuale. Agli italiani veniva fatta balenare la possibilità di accedere a una sorta di harem coloniale, con la precisa funzione di rendere desiderabile il trasferimento nelle colonie. La retorica coloniale fu impregnata di metafore sessuali: si parlò di penetrazione nei nuovi territori, di selvaggi effeminati, dell'ardire dei soldati italiani di fronte alle bellezze native. Come sottolinea Labanca nelle memorie dei reduci d'Africa, la presenza femminile nativa assume una dimensione straordinaria: «le donne native costituirono un punto fermo dell'esperienza italiana» ${ }^{[8]}$, divenendo nel ricordo il lato affascinante, perturbante e contraddittorio dell'esperienza coloniale. 
Letteratura e cinema ebbero un ruolo centrale nella costruzione di questo immaginario; esaltando la bellezza delle indigene, mettevano in scena il desiderio di conquista sessuale della donna africana, presentata come femmina autentica, non corrotta dalla civiltà, al contrario dell'europea emancipata, come emerge da una delle canzoni più popolari del nostro paese, Faccetta nera ${ }^{[9]}$ L'erotizzazione delle donne africane si tradusse, nella realtà, nel loro impiego come oggetti sessuali a disposizione di militari e civili. Le relazioni tra donne africane e uomini italiani furono talmente frequenti che venne coniato, per indicarle, uno specifico eufemismo: rapporti di madamato, espressione che indicava la «relazione temporanea, ma non occasionale, tra un cittadino e una suddita indigena»[10] e serviva a coprire lo sfruttamento sessuale e domestico delle africane. Si noti che il termine madama conteneva una connotazione spregiativa, dato che derivava dall'appellativo usato in Italia e Francia per indicare le tenutarie dei bordelli. Nel primo periodo della colonizzazione, gli ufficiali furono incoraggiati a "prender madama" sia come misura di protezione dalle malattie veneree, sia per preservarne il prestigio, evitando che condividessero le prostitute con i subordinati italiani ed eritrei, dato che i postriboli non erano segregati. $\stackrel{11]}{ }$ Negli anni successivi alla sconfitta di Adua (1896), nei quali il governo dell'Eritrea era affidato a Ferdinando Martini, il concubinaggio interrazziale conobbe un grande sviluppo, anche se in seguito furono fatti alcuni tentativi, per lo più infruttuosi, per limitarlo.

Le cose cambiarono nella seconda metà degli anni Trenta, quando, dopo la conquista dell'Etiopia e la proclamazione dell'impero, il regime fascista operò una decisa inversione di rotta, vietando le relazioni tra italiani e africane e mettendo al centro della comunicazione il tema della lotta al meticciato. Archiviate le immagini della Venere nera, la propaganda fascista si impegnò nella diffusione di rappresentazioni di tipo etnografico, che ponevano in risalto caratteristiche fisiche e psichiche degli africani, ritenute segni di inferiorità, allo scopo di riaffermare la superiorità degli europei e la legittimità della loro colonizzazione. Sul piano giuridico, vennero implementati dei dispositivi miranti a controllare il comportamento di italiani e "sudditi" per riaffermare il prestigio razziale dei primi. Furono vietate le relazioni coniugali ed extraconiugali tra "razze" diverse, proibita la legittimazione e l'adozione di figli nati dall'unione di "cittadini" con "suddite", instaurata una capillare segregazione razziale. I "meticci" furono ricacciati nella comunità indigena e ogni istituzione precedentemente creata per la loro assistenza fu posta fuori legge. Tali misure ottemperavano al desiderio di rafforzare la piramide etnica senza consentire, al suo interno, alcuna zona grigia, per garantire alla "razza italiana" un posto di spicco tra i colonizzatori. Le conseguenze che ebbero sulla vita e i vissuti delle donne africane e dei loro figli sono raccontate, con accenti toccanti, da Erminia Dell'Oro in L'abbandono. ${ }^{[12]}$

Durante la dominazione italiana, le donne africane subirono molti tipi di violenze. Da un lato furono vittime del conflitto come parte della popolazione civile, dall'altro furono vittime di violenze specifiche, derivanti dalla combinazione di una triplice stigmatizzazione: di razza, di classe, di genere. Tali violenze non sono state facili da documentare data la scarsità e la reticenza delle testimonianze e il silenzio degli archivi. Tuttavia, la ricerca storica ha negli ultimi decenni scavato nei documenti e nelle pagine della memorialistica registrando e descrivendo una serie di casi che, secondo la lezione di Ginzburg $\stackrel{[13]}{ }$, sono interpretabili come tracce di una realtà ben più vasta, dato che indicano concordemente che le pratiche di sopruso e violenza erano largamente diffuse e restavano il più delle volte impunite. $\stackrel{[14]}{ }$

\section{Un mito per rimuovere il passato coloniale: Italiani brava gente}

Un silenzio assordante - per riprendere il titolo di un bel libro di Patrizia Romito[15] sull'occultamento della violenza a donne e bambini - circonda ancora oggi in Italia le violenze 
subite dalle donne africane. Da un lato, la documentazione delle loro sofferenze risente del generale ritardo dell'indagine storica sulle vicende coloniali italiane, causato dalla volontà politica di non fare i conti con il passato. Dall'altro, pochissime sono le ricerche sulla storia delle donne in Africa prima, durante, dopo la colonizzazione. Sul periodo del colonialismo italiano, il silenzio è quasi assoluto: la voce delle africane non compare mai né nei documenti ufficiali, dai quali è peraltro assente anche la voce delle italiane, né nelle testimonianze orali raccolte dagli storici, che, con l'eccezione di Barrera, hanno intervistato solo uomini.

La ragione più profonda del silenzio può però essere individuata nel fatto che la memoria del passato coloniale è stata permeata dal mito auto-assolutorio degli Italiani brava gente, che descrive gli italiani come colonizzatori tolleranti, umani, incapaci di comportamenti crudeli. Si tratta, ancora una volta, di un mito maschile, dato che è intorno all'uomo italiano, soldato e colono, che ruota tutto l'immaginario coloniale. Il mito, nato nelle prime fasi dell'avventura coloniale, è poi entrato stabilmente nell'immagine nazionale, arricchendosi via via di nuove componenti, relative ai comportamenti dei militari italiani durante il secondo conflitto mondiale e della popolazione civile verso gli ebrei nel periodo dell'occupazione nazista. Una componente, quest'ultima, che ha per anni consentito di sottovalutare il fatto che il governo italiano avesse promulgato, e il paese sostanzialmente accettato, le vergognose leggi razziali riguardanti i popoli delle colonie e la minoranza ebraica. ${ }^{[16]}$

Nel secondo dopoguerra, il mito degli Italiani brava gente si è rafforzato perché funzionale alla restaurazione dell'immagine nazionale italiana. La peculiarità italiana non deriva comunque dalla presenza del mito, dato che altri colonialismi, come quello portoghese per esempio, sono stati accompagnati da analoghi strumenti di autoassoluzione, ma dal fatto che nel nostro paese la rimozione perdura ancora e, anche dopo molti anni, l'opinione pubblica resta nella sua maggioranza impermeabile agli inviti a riflettere criticamente sul passato.

Negli anni Novanta dello scorso secolo il nostro paese ha reagito ai flussi migratori che lo hanno investito in modo massiccio e improvviso ricorrendo al patrimonio coloniale di credenze e atteggiamenti mai stato posto in discussione. Si è così creato un cortocircuito tra l'antica immagine coloniale dell'indigeno e quella recente dell'immigrato. ${ }^{[17]}$ La persistente vitalità del patrimonio coloniale è stata anche illustrata da una ricerca di Paola Tabet che, analizzando migliaia di temi scritti da alunni delle scuole elementari e medie, ha messo in luce come i bambini nati negli anni Ottanta riattualizzassero nei confronti degli immigrati atteggiamenti e vissuti desunti dall'immaginario coloniale 118$]$.

Il mito degli Italiani brava gente continua ancor oggi a produrre i suoi effetti deleteri, rendendo opaca la connessione tra passato e presente, tra il comportamento attuale verso i migranti e la mancata assunzione di responsabilità per le sofferenze inflitte alle vittime della colonizzazione. Ieri la credenza autoassolutoria celava i crimini coloniali; oggi contribuisce a nascondere quello che succede alle persone che tentano di approdare nel nostro paese, rendendo invisibili le morti in mare e le condizioni inumane dei cosiddetti centri di accoglienza. Tra i nessi oscurati vi è il legame tra gli abusi inflitti alle africane durante la colonizzazione e gli abusi inflitti oggi sulle strade italiane, dove donne e ragazze minorenni sono costrette a prostituirsi in una situazione di moderna schiavitù. Molte di loro provengono dall'Africa, portate in Europa dall'aspirazione a una vita migliore, ma anche da meccanismi di inganno e coercizione. La loro presenza riproduce le "relazioni pericolose" dello scenario coloniale in tutta la loro asimmetria di genere e di "razza": ancora una volta le donne africane diventano strumento di soddisfazione dei "bisogni" degli uomini italiani, in un contesto che perpetua processi di oggettivazione sessuale. 
Allo scopo di capire i meccanismi di rimozione dell'esperienza coloniale italiana, la collega Giovanna Leone dell'Università La Sapienza di Roma, ha aperto da più di un decennio un cantiere di ricerca per indagare una possibile causa dei "vuoti di memoria" che caratterizzano la popolazione italiana rispetto al passato coloniale. Leone ipotizza che un ruolo importante possa essere giocato dal modo in cui gli avvenimenti storici relativi all'esperienza coloniale sono insegnati alle nuove generazioni durante l'iter scolastico. Con alcuni colleghi la studiosa ha quindi analizzato le descrizioni dell'invasione dell'Etiopia contenute nei libri di testo usati nelle scuole superiori, ponendo in luce come essi dedichino solitamente poco spazio alla descrizione degli orrori commessi dagli italiani. Per esempio, in genere, l'aggressione e la conquista dell'Etiopia sono presentate in un breve paragrafo, inserito nel capitolo sul fascismo, illustrato da immagini d'epoca, prodotte dalla stessa propaganda del regime. Solo pochi testi descrivono i crimini di guerra in modo chiaro e dettagliato, senza impiegare un linguaggio elusivo. $\frac{[19]}{[1}$

Altri lavori hanno esplorato il ruolo delle narrazioni intergenerazionali, che possono contribuire ai processi di riconciliazione tra gruppi segnati da pesanti conflitti; ${ }^{[20]}$ in altri ancora sono stati impiegati metodi sperimentali, raccontando, mediante modalità chiare (parresia) o evasive, a gruppi di studenti gli avvenimenti principali della conquista dell'Etiopia, e chiedendo loro di esprimere le emozioni provate durante la lettura. I risultati hanno mostrato che solo un'informazione chiara sui crimini italiani cambia significativamente il vissuto emozionale di chi ascolta, promuovendo sentimenti di disagio e tristezza e diminuendo l'indifferenza; allo stesso modo, solo un'informazione franca e non reticente porta i giovani a esprimere giudizi morali sui crimini del passato e a esperire sentimenti di colpa collettiva. ${ }^{[21]}$

\section{Razzismo e forme attuali del fascismo}

Partiamo da una premessa. Umberto Eco descrive nel 2017 le quattordici caratteristiche di quello che, a suo parere, è l'Ur-Fascismo o il Fascismo Eterno, avvertendo che tali caratteristiche non possono essere irreggimentate in un sistema, che alcune si contraddicono tra loro e molte sono tipiche anche di altre forme di dispotismo o fanatismo. È però sufficiente, secondo Eco, che una di loro sia presente "per far coagulare una nebulosa fascista". 22$]$ Se passiamo in rassegna tali caratteristiche, scopriamo che la maggior parte è presente negli atteggiamenti e nei comportamenti di quello che potremmo chiamare fascismo contemporaneo. Sicuramente l'irrazionalismo, il rifiuto del modernismo e della critica, il razzismo, il machismo, l'elitismo popolare, il populismo qualitativo, la neolingua.

Lo schema proposto da Eco è utile perché aiuta a reperire anche nei fenomeni contemporanei delle linee di tendenza che ancorano tali fenomeni alla storia passata. Per capire a pieno tali fenomeni, però, occorre valutarne non solo gli aspetti di continuità, ma anche gli aspetti di novità e specificità. Le contraddizioni che segnano il presente nascono da trasformazioni economiche (il tramonto del paradigma fordista, la finanziarizzazione dell'economia, l'affermarsi del capitalismo oligarchico, la vittoria dell'individualizzazione nei rapporti di lavoro); culturali (l'egemonia del neoliberismo, si veda a questo proposito il recente libro di Marco D'Eramo ${ }^{[23]}$ ); politiche (l'affermarsi sullo scenario internazionale di nuove potenze in grado di sfidare la supremazia statunitense) e sociali (la crescita delle disuguaglianze, la proletarizzazione delle classi medie dei paesi occidentali, lo strapotere dei grandi ricchi), che vanno esaminate in profondità per capire quale sia il loro ruolo nel risorgere di atteggiamenti e comportamenti che possono essere definiti fascisti.

Le forme contemporanee di fascismo scaturiscono da profondi stati di frustrazione individuali e collettivi provati da settori sociali che percepiscono di essere penalizzati dalla globalizzazione e di 
star perdendo l'antico e comodo "dividendo coloniale" a favore delle classi medie dei paesi emergenti. Parti di tali settori cercano un'illusoria, ma rassicurante identità sociale basata sulla nazionalità, l'etnia, la religione, un'identità che ricorda il nazionalismo tribale di cui parla Hannah Arendt nelle Origini del totalitarismo ${ }^{[24]}$, che consegna alla comunità di sangue i criteri per includere o escludere dalla cittadinanza e nasce da vissuti di inadeguatezza e senso di inferiorità, a cui si tenta di reagire con lo strumento del razzismo. E, in questo percorso, ritrovano pensieri, sentimenti, comportamenti che richiamano il fascismo.

Note:

[1] Per fare un solo esempio, un recente rapporto della Federal Reserve Bank di New York, firmato da Kristian Blickle e pubblicato il 20 maggio 2020, Pandemics change cities: Municipal spending and voter extremism in Germany, 1918-1933, suggerisce che l'epidemia di spagnola verificatasi alla fine della prima guerra mondiale abbia avuto in Germania un ruolo importante nell'allontanare, all'inizio degli anni Trenta, l'elettorato tedesco dalla democrazia di Weimar e nello spingerlo verso i partiti estremisti, tra cui quello nazionalsocialista. Secondo il rapporto, il fenomeno sarebbe stato imputabile alle difficoltà finanziarie che avevano impedito alle amministrazioni locali di prestare assistenza in modo efficace alla popolazione.

${ }^{[2]}$ D. Capozza, C. Volpato, Le intuizioni psicosociali di Hitler: Un'analisi del Mein Kampf, Patron, Bologna 2004

[3] C. Volpato (2013). Negare l'altro. La deumanizzazione e le sue forme, in "Psicoterapia e Scienze Umane", n. 47(2), 2013, pp. 311-328. Si veda anche: C. Volpato, F. Durante, A. Gabbiadini, L. Andrighetto, S. Mari, Picturing the other: Targets of delegitimization across time, in "International Journal of Conflict and Violence", n. 4(2), 2010, pp. 269-287.

${ }^{[4]}$ R. R. Valtorta, C. Baldissarri, L. Andrighetto, C. Volpato "We are the virus": Outgroup and ingroup biologization in the time of Covid-19. Manoscritto inviato per pubblicazione.

${ }^{[5]}$ Si veda, ad esempio, La Repubblica, 16 novembre 2020.

[6] C. Ghezzi, Famiglia, patria e impero: Per una storia della donna italiana in colonia, in "Studi Piacentini”, n. 30, 2001, pp. 91-129.

${ }^{[7]}$ B. Mussolini, Scritti e discorsi. X: Scritti e discorsi dell'impero (novembre 1935 - 4 novembre 1936). Hoepli, Milano 1936, p. 31.

${ }^{[8]}$ N. Labanca, Una guerra per l'impero. Memorie della campagna d'Etiopia 1935-36, Il Mulino, Bologna 2005, p. 265.

${ }^{[9]}$ L. Ellena, Mascolinità e immaginario nazionale nel cinema italiano degli anni Trenta, In S. Bellassai e M. Malatesta (a cura di), Genere e mascolinità. Uno sguardo storico Bulzoni, Rom 2000, pp. 243- 264.

$[10]$ B. Sòrgoni, Parole e corpi. Antropologia, discorso giuridico e politiche sessuali interrazziali nella colonia Eritrea (1890-1941), Liguori, Napoli 1998, p. 74. Si veda anche: G. Campassi, Il 
madamato in A.O.: relazioni tra italiani e indigene come forma di aggressione coloniale in "Miscellanea di Storia delle Esplorazioni”, XII, 1983, pp. 219-258.

${ }^{[11]}$ G. Barrera, Sessualità e segregazione nelle terre dell'Impero, in R. Bottoni (a cura di), L'Impero fascista. Italia ed Etiopia (1935-1941), Il Mulino, Bologna 2008, pp. 393-414.

${ }^{[12]}$ E. Dell'Oro, L'abbandono, Einaudi, Torino 1991.

${ }^{[13]}$ C. Ginzburg, Spie. Radici di un paradigma indiziario, in A. Gargani (a cura di), Crisi della ragione. Nuovi modelli nel rapporto tra sapere e attività umane Einaudi, Torino 1979, pp. 57-106.

${ }^{114]}$ G. Barrera, Dangerous liaisons: Colonial concubinage in Eritrea (1890 -1941), in Program of African Studies Working Papers, 1, Northwestern University, Evanston 1996. N. Poidimani, «Faccetta nera»: i crimini sessuali del colonialismo fascista nel Corno d'Africa, in L. Borgomaneri (a cura di), Crimini di guerra. Il mito del bravo italiano tra repressione del ribellismo e guerra ai civili nei territori occupati, Guerini e associati, Milano 2006, pp. 33-62. N. Poidimani, Difendere la "razza". Identità razziale e politiche sessuali nel progetto imperiale di Mussolini, Sensibili alle foglie, Roma 2009. B. Sòrgoni, Etnografia e colonialismo. L'Eritrea e l'Etiopia di Alberto Pollera (1873-1939), Bollati Boringhieri, Torino 2001. G. Stefani, Colonia per maschi. Italiani in Africa Orientale: una storia di genere, Ombre Corte, Verona 2007. C. Volpato, La violenza contro le donne nelle colonie italiane. Prospettive psicosociali di analisi, in "Deportate, esuli, profughe. Rivista telematica di studi sulla memoria femminile", n. 10, 2009, pp. 110-131. C. Volpato, A. Gabbiadini, La maschilità nelle colonie italiane, in S. Magaraggia e D. Cherubini (a cura di), Uomini contro le donne? Le radici della violenza maschile, UTET, Torino 2013, pp. 103-127.

${ }^{[15]}$ P. Romito, Un silenzio assordante. La violenza occultata su donne e minori, Franco Angeli, Milano 2005.

[16] D. Bidussa, Il mito del bravo italiano, Il Saggiatore, Milano 1994. A. Del Boca, Italiani, brava gente? Un mito duro a morire, Neri Pozza, Vicenza 2005.

[17] P. Blanchard, N. Bancel, De l'indigène à l'immigré, Gallimard, Parigi, 1998.

${ }^{[18]}$ P. Tabet, La pelle giusta. Einaudi, Torino 1997.

${ }^{[19]}$ G. Leone, T. Mastrovito, Learning about our shameful past: A socio-psychological analysis of present-day historical narratives of Italian colonial wars, in "International Journal of Conflict and Violence", 4, 1, 2010, pp. 11-27.

$\stackrel{[20]}{\mathrm{G}}$. Leone, Se gli inumani siamo noi. Una riflessione sulla narrazione intergenerazionale dei crimini di guerra del proprio gruppo, in "Rivista Internazionale di Filosofia e Psicologia", 2, 2, 2011, pp. 131-147.

${ }^{[21]}$ G. Leone, When history teaching turns into Parrhesia: The case of Italian colonial crimes, in C. Psaltis, M. Carretero e S. Cehajic-Clancy (a cura di), History education and conflict transformation. Social psychology theories, history teaching and reconciliation, Palgrave Macmillan, Cham 2017, pp. 147-168. G. Leone, R. D’Ambrosio, F. D’Errico, S. Migliorisi, Facing the unknown crimes of older generations: Emotional and cognitive reactions of young Italian student reading an historical text on colonial invasion of Ethiopia, in "International Journal of Intercultural Relations", n. 62, 2018, pp. 55-67. G. Leone, M. Giner-Sorolla, S. Migliorisi, I. Sessa, It's time to be ashamed! Reactions to the breaking of a long-lasting self-censorship on ingroup war crimes, in "TPM", n. 25, 
2018, pp. 519-535. G. Leone, M. Sarrica, Challenging the myth of Italians as 'Good Fellows': Is clarity about ingroup crimes the best choice when narrating the war to its perpetrators'

descendants?, in "Papers on Social Representations", n. 21(2), 2012, pp. 11-1. G. Leone, M.

Sarrica, Making room for negative emotions about the national past: An explorative study of effects of parrhesia on Italian colonial crimes, in "International Journal of Intercultural Relations", n. 43, 2014, pp. 126-138. G. Leone, M. Sarrica, The making of a civic discourse on controversial

historical past: From denial to parrhesia in "ESSACHESS - Journal for Communication Studies", n. 10, 2017, pp. 33-53.

${ }^{[22]}$ U. Eco, Il fascismo eterno. La nave di Teseo, Milano 2017, p. 23.

${ }^{[23]}$ M. D’Eramo, Dominio. La guerra invisibile dei potenti contro i sudditi. Feltrinelli, Milano 2020.

${ }^{[24]}$ H. Arendt, The origins of totalitarianism. Harcourt, Brace \& World, New York 1951. Trad. it. Le origini del totalitarismo. Edizioni di Comunità, Milano 1967. 